\title{
BUSINESS
}

\section{Quantum leap of faith}

\section{A Canadian company says it is the first to bring a quantum computer to market but, as Geoff Brumfiel reports, not everyone is buying into the approach.}

$T$ he public debut of what is being billed as the world's first commercial quantum computer was a low-key affair. And so it should have been, say critics.

D-Wave Systems, a private company based in British Columbia, Canada, launched its machine last month at the Computer History Museum in Mountain View, California. Geordie Rose, D-Wave's founder and chief technology officer, tried to persuade an audience of scientists, journalists and entrepreneurs that the machine called Orion - could be used to solve awkward problems such as how to seat a wedding party to minimize friction between the guests.

"We want to build a machine that cannot be bettered by any machine that obeys the laws of physics," says Rose, whose company has adopted a rapid-fire approach to product development. "The philosophy behind our design is build the thing as fast, as dirty and as quickly as you can."

Quantum specialists greeted the debut with scepticism, however. In the run-up to the announcement, D-Wave failed to convince outside researchers that its machine actually works in a quantum regime, says Scott Aaronson, a computer scientist at the University of Waterloo in Ontario, Canada, who writes programs for quantum computers. And even if it does, he says, its 16 quantum bits (qubits) are insufficient to outperform conventional computers: "Even if it worked perfectly, it's still completely useless from an industrial perspective."

Others are a little more sanguine. The approach of going ahead and building the machine without much in the way of proof that it will work is risky, but might bear fruit, says Seth Lloyd, a mechanical engineer at the Massachusetts Institute of Technology in Cambridge who specializes in quantum computing.

Where conventional computers rely on electrons to encode 'bits' at one or zero, quantum computers encode their information in the different quantum states of electrons, photons or atomic nuclei. Relationships between these states are known as 'superposition' and 'entanglement' in the quantum world, and theorists think that they could allow the machines to crunch numbers much more quickly than their conventional counterparts. In particular, they should be able to identify the factors of large

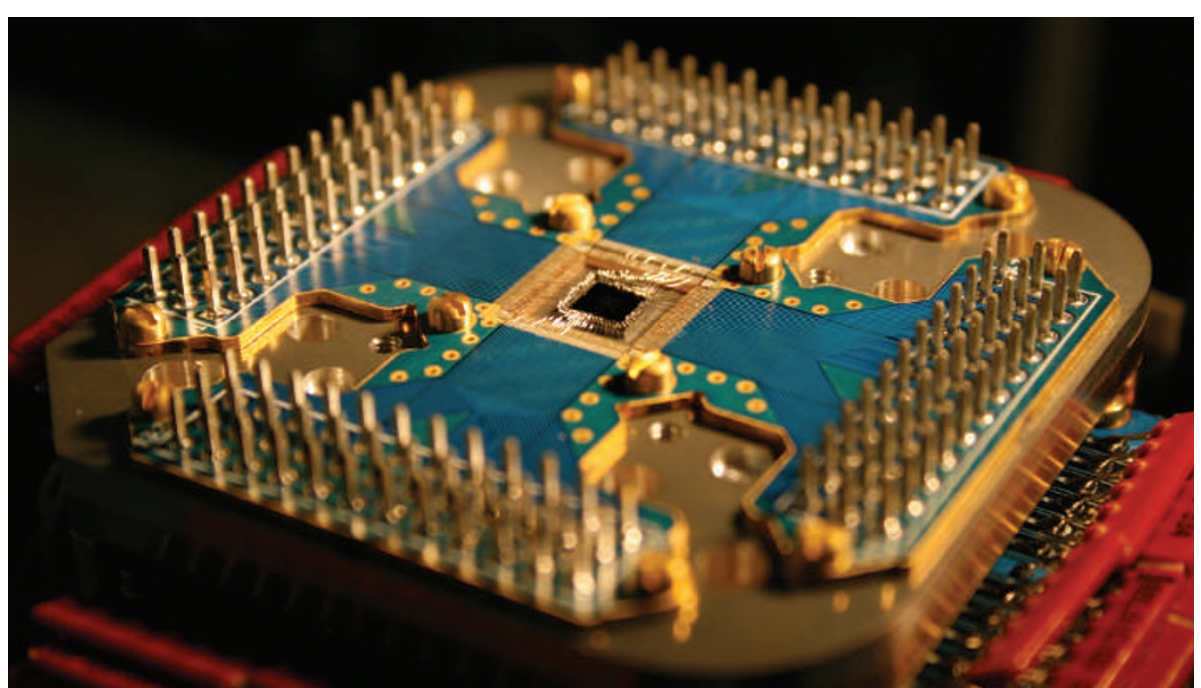

D-Wave's 16-qubit processor uses an untested approach to solve complex problems.

numbers much faster, making them valuable for code-breaking. They might also be able to solve a simple problem from a vast set of possibilities, as the example of the wedding seating plan sought to demonstrate.

Major computing firms such as HewlettPackard and IBM have small research groups working away at quantum computation, but see it as a long-term prospect. The main challenge is to build a machine that can set, maintain and read quantum states without being overwhelmed by surrounding noise, specialists say. Control systems need to be exquisitely sensitive, and the very act of writing or reading can disrupt their operation. Most existing quantum computers look like physics experiments, and contain very few actual qubits.

D-Wave's approach is known as adiabatic quantum computing. It uses a superconducting microchip to create spinning loops of current, which it says act as qubits, spinning in both directions at once. The qubits then entangle together, putting the entire machine in a 'quantum ground state'. By slowly changing the magnetic fields around the qubits, the computer's operator can then change the computer's entanglement, moving it into a new ground state. Reading this state can, according to the designers, reveal a solution to a complex prob- lem without the need to directly read the state of individual qubits.

Aaronson says, however, that external noise could still be a problem for the machine. $\mathrm{He}$ also notes that the company has provided no public evidence that it has its system under control - or that its qubits are entangled correctly. According to Lloyd, researchers are still debating whether the entire approach is of any use: "Nobody knows exactly whether adiabatic quantum computing will work or not."

\section{No rush to publish}

But Rose says that the company's scientists are satisfied that the system is indeed operating properly - and that the objective is to try something out and see whether it works, rather than to pin down exactly how it does so. "I understand the scientific community would like to see publications coming from us," he says. "But this project has never been about science, it's about building a machine."

And D-Wave has managed to persuade key investors to stick with the concept, raising some US\$38 million since it was founded in 1999 , half from the Canadian government and half from venture-capital firms, including Draper Fisher Jurvetson of Menlo Park, California. But even Rose still sees success as an outside bet, and Lloyd shares his reservations. "Investing in something like D-Wave is only for someone who wants to take risks," Lloyd says. "Big risks." 